\title{
XLVI. On quaternions; or on a new system of imaginaries in algebra
}

\author{
Sir William Rowan Hamilton LL.D. V.P.R.I.A. F.R.A.S.
}

To cite this article: Sir William Rowan Hamilton LL.D. V.P.R.I.A. F.R.A.S. (1847) XLVI. On quaternions; or on a new system of imaginaries in algebra, Philosophical Magazine Series 3, 31:208, 278-293, DOI: 10.1080/14786444708562643

To link to this article: http://dx.doi.org/10.1080/14786444708562643

曲 Published online: 30 Apr 2009.

Submit your article to this journal $[\pi$

Џ Article views: 4

Q View related articles $\square$

Citing articles: 1 View citing articles 5 


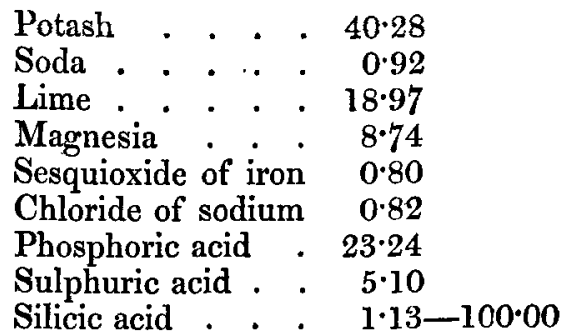

The preceding analyses furnish a new confirmation of the fact first observed by De Saussure, namely, that the largest amount of mineral constituents is deposited in those parts of the plant in which the process of assimilation appears to be most active. While the ash left by the root, stem, fruit and seed did not exceed from 3 to 4 per cent., the leaves left not less than 13 per cent. of fixed residue on incineration.

Regarding the composition of the different ashes, the great amount of carbonic acid found in the ashes of the root, the stem, and the fruit is at once obvious; proving that not only the fruit, but also the roots and stem, contain a large quantity of organic acids.

From the composition of the ashes of the root, the stem, and the leaves, the orange-tree belongs decidedly to the lime plants. In these three ashes the joint amount of lime and magnesia exceeds the quantity of the rest of the mineral constituents. In the ashes of the fruit and seed, however, the alkalies are as prevalent as they have been fourd in analogous cases. The amount of phosphoric acid (23.24) in the ash of the seed is considerable, as might be expected, still it is inferior to the quantities (34.81 and 42.02) which Mr. Souchay found on analysing the seeds of the citron (Citrus Medica) and quince-trees (Pyrus Cydonia). Nevertheless the ash of the orange-seed is very analogous in composition to the ashes of the last-mentioned sceds, as may be easily seen on comparing their analysis*.

XLVI. On Quaternions; or on a New System of Imaginaries in Algebra. By Sir William Rowan Hamilton, LL.D., V.P.R.I.A., F.R.A.S., Corresponding Member of the Institute of France, \&c., Andreves' Professor of Astronomy in the University of Dublin, and Royal Astronomer of Ireland.

[Continued from p. 219.]

37. RESUMING now the quaternion form of the equation of the ellipsoid,

$$
\begin{aligned}
& (\alpha \rho+\rho \alpha)^{2}-(\beta p-\rho \beta)^{2}=1, . \quad . \quad \\
& * \text { Liebig's Annals, liv. p. } 343 .
\end{aligned}
$$


and making

$$
\alpha+\beta=\frac{1}{\iota^{2}-x^{2}}, \quad \alpha-\beta=\frac{x}{\iota^{2}-x^{2}}, \quad . . .
$$

and

$$
\frac{p+p x}{t^{2}-x^{2}}=Q, \quad \frac{p l+x p}{\iota^{2}-x^{2}}=Q^{\prime}, \quad . \quad . \quad \cdot
$$

the two linear factors of the first member of the equation (1.) become the two conjugate quaternions $Q$ and $Q^{\prime}$, so that the equation itself becomes

$$
Q Q^{\prime}=1 \text {. . . . . . . }
$$

But by articles 19 and 20 (Phil. Mag. for July 1846), the product of any two conjugate quaternions is equal to the square of their common tensor; this common tensor of the two quaternions $Q$ and $Q^{\prime}$ is therefore equal to unity. Using, therefore, as in those articles, the letter ' $T$ as the characteristic of the operation of taking the tensor of a quaternion, the equation of the ellipsoid reduces itself to the form

$$
\mathrm{TQ}=1 ; \text {. . . . . . . }
$$

or, substituting for $Q$ its expression (3.),

$$
\mathrm{T}\left(\frac{p+p x}{\imath^{2}-x^{2}}\right)=1 ; . . . . . .
$$

which latter form might also have been obtained, by the substitutions (2.), from the equation (3.) of the 30th article (Phil. Mag., June 1847), namely from the following*:

$$
\boldsymbol{T}(\alpha \rho+\rho \alpha+\beta \rho-\rho \beta)=1 . \quad . \quad . \quad .
$$

38. In the geometrical construction or generation of the ellipsoid, which was assigned in the preceding articles of this paper (see the Numbers of the Philosophical Magazine for June and September 1847), the significations of some of the recent symbols are the following. The two constant vectors 1 and $x$ may be regarded as denoting, respectively, (in lengths and in directions, ) the two sides of the generating triangle $A B C$, which are drawn from the centre $c$ of the auxiliary and diacentric sphere, to the fixed superficial point $\mathbf{B}$ of the ellip. soid, and to the centre $\Delta$ of the same ellipsoid; the third side of the triangle, or the vector from $\mathrm{A}$ to $\mathrm{B}$, being therefore denoted (in length and in direction) by $1-x:$ while $\rho$ is the radius vector of the ellipsoid, drawn from the centre $A$ to a

* See equation (35.) of the Abstract in the Proceedings of the Royal Irish Academy for July 1846. The equation of the ellipsoid marked (1.) in article 37 of the present paper, was communicated to the Academy in December 1845, and is numbered (21.) in the Proceedings of that date. 
variable point $E$ of the surface; so that the constant vector $1-x$ is, by the construction, a particular value of this variable vector $p$. The vector from $\mathrm{A}$ to $\mathrm{c}$, being the opposite of that from $c$ to $A$, is denoted by $-x$; and if $D$ be still the same auxiliary point on the surface of the auxiliary sphere, which was denoted by the same letter in the account already printed of the construction, then the vector from $\mathrm{C}$ to $\mathrm{D}$, which may be regarded as heing (in a sense to be hereafter more fully considered) the reflexion of $-x$ with respect to $\rho$, is $=-p x \rho^{-1}$; and consequently the vector from $D$ to $B$ is $=1+\exp ^{-1}$. The lengths of the two straight lines $\mathrm{BD}$, and $\mathrm{AE}$, are therefore respectively denoted by the two tensors, $T\left(b+p x_{p}{ }^{-1}\right)$ and $T_{p}$; and the rectangle under those two lines is represented by the product of these two tensors, that is by the tensor of the product, or by $T(p+p x)$. But by the fundamental equality of the lengths of the diagonals, $\mathbf{A E}, \mathbf{B D}^{\prime}$, of the plane quadrilateral $\mathrm{ABED}^{\prime}$ in the construction, this rectangle under $\mathrm{BD}$ and $A E$ is equal to the constant rectangle under $\mathbf{B D}$ and $\mathbf{B D}^{\prime}$, that is under the whole secant and its external part, or to the square on the tangent from $B$, if the point $B$ be supposed external to the auxiliary sphere, which has its centre at $c$, and passes through $\mathrm{D}, \mathrm{D}^{\prime}$, and $\mathrm{A}$. Thus $\mathrm{T}\left(t_{p}+p x\right)$ is equal to $\left(\mathrm{T}_{1}\right)^{2}-(\mathrm{T} x)^{2}$, or to $x^{2}-t^{2}$, which difference is here a positive scalar, because it is supposed that $\mathrm{CB}$ is longer than $\mathrm{CA}_{\mathrm{A}}$, or that

$$
\mathrm{T}_{1}>\mathrm{T}_{\boldsymbol{x}} \text {; . . . . . . }
$$

and the quaternion equation (6.) of the ellipsoid reproduces itself, as a result of the geometrical construction, under the slightly simplified form*

$$
T(\iota p+\rho x)=x^{2}-\imath^{2} . \quad . \quad . \quad . \quad .
$$

And to verify that this equation relative to $\rho$ is satisfied (as we have seen that it ought to be) by the particular value

$$
\rho=\imath-x, \quad \cdot . \cdot \text {. . }
$$

which corresponds to the particular position $B$ of the variable point $\mathbf{E}$ on the surface of the ellipsoid, we have only to observe that, identically,

$$
\begin{aligned}
& r(b-x)+(\imath-x) x=i^{2}-b x+i x-x^{2} \\
& =b^{2}-x^{2}=-\left(x^{2}-\imath^{2}\right)
\end{aligned}
$$

and that (by article 19) the tensor of a negative scalar is equal to the positive opposite thereof.

39. The foregoing article contains a sufficiently simple

* See the Proceedings of the Royal Irish Academy for July 1846, equation (44.). 
process for the retranslation of the geometrical construction* of the ellipsoid described in article 31 , into the language of the calculus of quaternions, from which the construction itself had been originally derived, in the manner stated in the 30th article of this paper. Yet it may not seem obvious to readers unfamiliar with this calculus, why the expression $-\rho x p^{-1}$ was taken, in that foregoing article 38 , as one denoting, in length and in direction, that radius of the auxiliary sphere which was drawn from $\mathrm{C}$ to $\mathrm{D}$; nor in what sense, and for what reason, this expression $-\rho x \rho^{-1}$ has been said to represent the reflexion of the vector $-x$ with respect to $\rho$. As a perfectly clear answer to each of these questions, or a distinct justification of each of the assumptions or assertions thus referred to, may not only be useful in connection with the present mode of considering the ellipsoid, but also may throw light on other applications of quaternions to the treatment of geometrical and physical problems, we shall not think it an irrelevant digression to enter here into some details respecting this expression- $\operatorname{pxp}^{-1}$, and respecting the ways in which it may present itself in calculations such as the foregoing. Let us therefore now denote by $\sigma$ the vector, whatever it may be, from $\mathrm{C}$ to $\mathrm{D}$ in the construction (c being still the centre of the sphere); and let us propose to find an expression for this sought vector $\sigma$, as a function of $\rho$ and of $x$, by the principles of the calculus of quaternions.

40. For this purpose we have first the equation between tensors,

$$
\mathrm{T} \sigma=\mathrm{T}_{\kappa} ; . . . \quad \cdot \quad \text { (11.) }
$$

which expresses that the two vectors $\sigma$ and $x$ are equally long, as being both radii of one common auxiliary sphere, namely those drawn from the centre $c$ to the points $D$ and $A$. And secondly, we have the equation

$$
\mathrm{V} \cdot(\sigma-x) \rho=0, \cdot \cdot \cdot \cdot \cdot \cdot
$$

where $\mathrm{V}$ is the characteristic of the operation of taking the vector of a quaternion; which equation expresses immediately that the product of the two vectors $\sigma-x$ and $\rho$ is scalar, and

* The brevity and novelty of this rule for constructing that important surface may perhaps justify the reprinting it here. It was as follows : From a fixed point $\mathrm{A}$ on the surface of a sphere, draw a variable chord $\mathrm{A} D$; let $D^{\prime}$ be the second point of intersection of the spheric surface with the secant $B D$, drawn to the variable extremity $D$ of this chord $A D$ from a fixed external point $B$; take the radius vector $\mathrm{AE}$ equal in length to $\mathrm{BD}^{\prime}$, and in direction either coincident with, or opposite to, the chord $A D$; the locus of the point $\mathrm{E}$, thus constructed, will be an ellipsoid, which will pass through the point $B$ (and will have its centre at A). See Proceedings of the Royal Irish Academy for July 1846. 
therefore that these two vector-factors are either exactly similar or exactly opposite in direction; since otherwise their product would be a quaternion, having always a vector part, although the scalar part of this quaternion-product $(\sigma-x) \rho$ might vanish, namely by the factors becoming perpendicular to each other. Such being the immediate and general signification of the equation (12.), the justification of our establishing it in the present question is derived from the consideration that the radius vector $\rho$, drawn from the centre $A$ to the surface $\mathbf{E}$ of the ellipsoid, has, by the construction, a direction either exactly similar or exactly opposite to the direction of that guide-chord of the auxiliary sphere which is drawn from $A$ to $D$, that is, from the end of the radius denoted by $x$ to the end of the radius denoted by $\sigma$. For, that the chord so drawn is properly denoted, in length and in direction, by the symbol $\sigma-x$, follows from principles respecting addition and subtraction of directed lines, which are indeed essential, but are not peculiar, to the geometrical applications of quaternions; had occurred, in various ways, to several independent inquirers, before quaternions (as products or quotients of directed lines in space) were thought of; and are now extensively received.

41. The two equations (11.) and (12.) are evidently both satisfied when we suppose $\sigma=x$; but because the point $D$ is in general different from $A$, we must endeavour to find another value of the vector $\sigma$, distinct from $x$, which shall satisfy the same two equations. Such a value, or expression, for this sought vector $\sigma$ may be found at once, so far as the equation (12.) is concerned, by observing that, in virtue of this latter equation, $\sigma-x$ must bear some scalar ratio to $\rho$, or must be equal to this vector $\rho$ multiplied by some scalar coefficient $x$, so that we may write

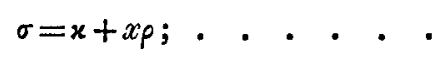

and then, on substituting this expression for $\sigma$ in the former equation (11.), we find that $x$ must satisfy the condition

$$
\mathrm{T}(x+x p)=\mathrm{T}_{x}, \ldots . . . .
$$

in which this sought coefficient $x$ is supposed to be some scalar different from zero, that is, in other words, some positive or negative number. Squaring both members of this last condition, and observing that by article 19 the square of the tensor of a vector is equal to the negative of the square of that vector, we find the new equation

$$
-(x+x \rho)^{2}=-x^{2} . \quad \text {. . . . . . }
$$

But also, generally, if $x$ and $\rho$ be vectors and $x$ a scalar,

$$
(x+x \rho)^{2}=x^{2}+x(x p+p x)+x^{2} p^{2} ;
$$


adding therefore $x^{2}$ to both members of (15.), dividing by $-x$, and then eliminating $x$ by (13.), which is done by merely changing $x p+x p^{2}$ to $\sigma \rho$, we find the equation

and finally

$$
\begin{aligned}
& \sigma \rho+\rho x=0 ; . . . . . \\
& \sigma=-\rho x \rho^{-1}: \text {. . . . . . }
\end{aligned}
$$

so that the expression already assigned for the vector from $\mathrm{C}$ to $D$, presents itself as the result of this analysis. And in fact the tensor of this expression (17.) is equal to $\mathrm{T} x$, by the general rule for the tensor of a product, or because $\left(-p x p^{-1}\right)^{2}$ $=\rho x \rho^{-1} \rho x \rho^{-1}=\rho x^{2} \rho^{-1}=x^{2}$, since $x^{2}$ is a (negative) scalar; while the product $(\sigma-x) \rho$, being $=-(x \rho+\rho x)$, is equal, by article 20 , to an expression of scalar form.

42. Conversely if, in any investigation conducted on the present principles, we meet with the expression $-\rho x \rho^{-1}$, we may perceive in the way just now mentioned, that it denotes a vector of which the square is equal to that of $x$; and that, if $x$ be subtracted from it, the remainder gives a scalar product when it is multiplied into $\rho$ : so that, if we denote this expression by $\sigma$, or establish the equation (17.), the equations (11.) and (12.) will then be satisfied, and the vector $\sigma$ will have the same length as $x$, while the directions of $\sigma-x$ and $p$ will be either exactly similar or exactly opposite to each other. We may therefore be thus led to regard, subject to this condition (17.) or (16.), the two vector-symbols $\sigma$ and $x$ as denoting, in length and in direction, two radii of one common sphere, such that the chord-line $\sigma-x$ connecting their ex tremities has the direction of the line $\rho$, or of that line reversed. Hence also, by the elementary property of a plane isosceles triangle, we may see that, under the same condition, the inclination of $\sigma$ to $\rho$ is equal to the inclination of $x$ to $-\rho$, or of $-x$ to $\rho$; in such a manner that the bisector of the external vertical angle of the isosceles triangle, or the bisector of the angle at the centre of the sphere between the two radii $\sigma$ and $-x$, is a new radius parallel to $\rho$, because it is parallel to the base of the triangle (ACD), or to the chord ( $A D)$ just now mentioned. And by conceiving a diameter of the sphere parallel to this chord, or to $\rho$, and supposing $-x$ to denote that reversed radius which coincides in situation with the radius $x$, but is drawn from the surface to the centre (that is, in the recent construction, from $\mathrm{A}$ to $\mathrm{c}$ ), while $\sigma$ is still drawn from centre to surface (from $\mathbf{C}$ to $\mathbf{D}$ ), we may be led to regard $\sigma$, or $-\rho \mathrm{p}^{-1}$, as the reflexion of $-x$ with respect to the diameter parallel to $\rho$, or simply with respect to $\rho$ itself, as was remarked in the 38 th article; since the vector-symbols $\rho, \sigma, \& c$. are supposed, 
in these calculations, to indicate indeed the lengths and directions, but not the situations, of the straight lines which they are employed to denote.

43. The same geometrical interpretation of the symbol - pxp $^{-1}$ may be obtained in several other ways, among which we shall specify the following. Whatever the lengths and directions of the two straight lines denoted by $\rho$ and $x$ may be, we may always conceive that the latter line, regarded as a vector, is or may be decomposed, by two different projections, into two partial or component vectors, $x^{\prime}$ and $x^{\prime \prime}$, of which one is parallel and the other is perpendicular to $\rho$; so that they satisfy respectively the equations of parallelism and perpendicularity (see article 21), and that we have consequently,

$$
x=x^{\prime}+x^{\prime \prime} ; \mathrm{V} \cdot x_{p}^{\prime}=0 ; \mathrm{S} \cdot x_{p}^{\prime \prime}=0 ; . \text {. }
$$

where $S$ is the characteristic of the operation of taking the scalar of a quaternion. The equation of parallelism gives $\rho x^{\prime}=x^{\prime} \rho$, and the equation of perpendicularity gives $p x^{\prime \prime}=-x^{\prime \prime} \rho$; hence the proposed expression $-\rho x \rho^{-1}$ resolves itself into the two parts,

$$
\left.\begin{array}{l}
-\rho x^{\prime} p^{-1}=-x^{\prime} \rho p^{-1}=-x^{\prime} ; \\
-\rho x^{\prime \prime} \rho^{-1}=+x^{\prime \prime} \rho p^{-1}=+x^{\prime \prime} ;
\end{array}\right\} . .
$$

so that we have, upon the whole,

$$
-\rho x \rho^{-1}=-\rho\left(x^{\prime}+x^{\prime \prime}\right) p^{-1}=-x^{\prime}+x^{\prime \prime} . \quad . \quad .
$$

'The part $-x^{\prime}$ of this last expression, which is parallel to $\rho$, is the same as the corresponding part of $-x$; but the part $+x^{\prime \prime}$, perpendicular to $\rho$, is the same with the corresponding part of $+x$, or is opposite to the corresponding part of $-x$; we may therefore be led by this process also to regard the expression (17.) as denoting the reflexion of the vector $-x$, with respect to the vector $\rho$, regarded as a reflecting line; and we see that the direction of $\rho$, or that of $-\rho$, is exactly intermediate between the two directions of $-x$ and $-p x p^{-1}$, or between those of $x$ and of $\rho \times p^{-1}$.

44. The equation (9.) of the ellipsoid, in article 38 , or the equation (4.) in article 37, may be more fully written thus:

$$
(p+p x)(p t+x p)=\left(x^{2}-t^{2}\right)^{2} \text {. . . . }
$$

And to express that we propose to cut this surface by any diametral plane, we may write the equation

$$
\varpi \rho+\rho \varpi=0, \cdot . \cdot \cdot \cdot \cdot
$$

where $\varpi$ denotes a vector to which that cutting plane is perpendicular : thus, if in particular, we change $\sigma$ to $x$, we find, for the corresponding plane through the centre, the equation 


$$
x p+p x=0, . . . . .
$$

which, when combined with (21.), gives

$$
\left(x^{2}-b^{2}\right)^{2}=(1-x) p \cdot p(1-x)=(1-x) p^{2}(1-x)=(1-x)^{2} p^{2},
$$

that is,

$$
p^{2}=\left(\frac{x^{2}-t^{2}}{1-x}\right)^{2} ; \quad \text {. . . . . }
$$

but this is the equation of a sphere concentric with the ellipsoid; therefore the diametral plane (23.) cuts the ellipsoid in a circle, or the plane itself is a cyclic plane. We see also that the vector $x$, as being perpendicular to this plane (23.), is one of the cyclic normals, or normals to planes of circular section; which agrees with the construction, since we saw, in article 36 , that the auxiliary or diacentric sphere, with centre $c$, touches one cyclic plane at the centre $A$ of the ellipsoid. The same construction shows that the other cyclic plane ought to be perpendicular to the vector 1 ; and accordingly the equation

$$
p+p t=0 . . . \cdot \text {. . }
$$

represents this second cyclic plane; for, when combined with the equation (21.) of the ellipsoid, it gives

$$
\left(x^{2}-i^{2}\right)^{2}=p(x-1) \cdot(x-1) p=p(x-1)^{2} p=(x-1)^{2} p^{2},
$$

and therefore conducts to the same equation (24.) of a concentric sphere as before; which sphere (24.) is thus seen to contain the intersection of the ellipsoid (21.) with the plane (25.), as well as that with the plane (23.). If we use the form (9.), we have only to observe that whether we change $\rho x$ to $-x p$, or ip to - $-p i$, we are conducted in each case to the following expression for the length of the radius vector of the ellipsoid, which agrees with the equation (24.):

$$
\mathrm{T}_{\rho}=\frac{x^{2}-1^{2}}{\mathrm{~T}(1-x)} \text {. . . . . . }
$$

And because $x^{2}-1^{2}$ denotes the square upon the tangent drawn to the auxiliary sphere from the external point $\mathrm{B}$, while $T(1-x)$ denotes the length of the side BA of the generating triangle, we see by this easy calculation with quaternions, as well as by the more purely geometrical reasoning which was alluded to, and partly stated, in the 36 th article, that the common radius of the two diametral and circular sections of the ellipsoid is equal to the straight line which was there called $\mathbf{B G}$, and which had the direction of $\mathbf{B A}$, while terminating, like it, on the surface of the auxiliary sphere; so that the two last lines $\mathrm{BA}$, and $\mathrm{BG}$, were connected with that sphere and with each other, in this or in the opposite order, as the whole se- 
cant and the external part. In fact, as the point $D$, in the construction approaches, in any direction, on the surface of the auxiliary sphere, to $A$, the point $\mathrm{D}^{\prime}$ approaches to $\mathrm{G}$; and $\mathrm{BD}^{\prime}$, and therefore also $\mathrm{AE}$, tends to become equal in length to $\mathrm{BG}$; while the direction of $A E$, being the same with that of $A D$, or opposite thereto, tends to become tangential to the sphere, or perpendicular to $\mathrm{AC}$ : the line $\mathrm{BG}$ is therefore equal to the radius of that diametral and circular section of the ellipsoid which is made by the plane that touches the auxiliary sphere at $\mathbf{A}$. And again, if we conceive the point $\mathbf{D}^{\prime}$ to revolve on the surface of the sphere from $\mathrm{G}$ to $\mathrm{G}$ again, in a plane perpendicular to $\mathrm{BC}$, then the lines $\mathrm{AD}$ and $\mathrm{AE}$ will revolve together in another plane parallel to that last mentioned, and perpendicular likewise to $\mathrm{BC}$; while the length of $\mathrm{AF}$ will be still equal to the same constant line $B G$ as before: which line is therefore found to be equal to the common radius of both the diametral and circular sections of the ellipsoid, whether as determined by the geometrical construction which the calculus of quaternions suggested, or immediately by that calculus itself.

45. We may write the equation (21.) of the-ellipsoid as follows :

$$
f(p)=1, \text {. . . . . }
$$

if we introduce a scalar function $f$ of the variable vector $\rho$, defined as follows :

$$
\left(x^{2}-r^{2}\right)^{2} f(p)=(u p+p x)(p b+x p)=s p^{2} b+r p x p+p x p b+p x^{2} p ;
$$

or thus, in virtue of article 20 ,

$$
\left(x^{2}-i^{2}\right)^{2} f(p)=\left(i^{2}+x^{2}\right) p^{2}+2 S \text {. spxp. . . }
$$

Let $\rho+\tau$ denote another vector from the centre to the surface of the same ellipsoid; we shall have, in like manner,

where

$$
f(p+\tau)=1 \text {, . . . . (29.) }
$$

$$
f(p+\tau)=f(p)+2 S . \nu \tau+f(\tau), . . .
$$

if we introduce a new vector symbol $\nu$, defined by the equation

$$
\left(x^{2}-r^{2}\right)^{2} y=\left(t^{2}+x^{2}\right) p+t p x+x f t ; \quad . \quad \text {. }
$$

because generally, for any two vectors $\rho$ and $\tau$,

$$
(\rho+\tau)^{2}=\rho^{2}+2 \mathrm{~S} \cdot \rho \tau+\tau^{2}, \ldots . . .
$$

and, for any four vectors, $i, x, \rho, \tau$,

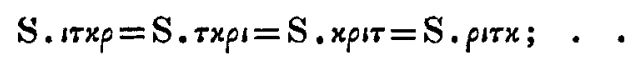

which last principle, respecting certain transpositions of vector symbols, as factors of a product under the sign S., shows, 
when combined with the equations (27.), (28.), and (31.), that we have also this simple relation:

$$
\text { S. } v \rho=1 \text {. }
$$

Subtracting (27.) from (29.), attending to (30.), changing $\tau$ to $T_{\tau} . U_{\tau}$, where $U$ is, as in article 19 , the characteristic of the operation of taling the versor of a quaternion (or of a vector), and dividing by $\mathrm{T}_{\tau}$, we find :

$$
0=\frac{f(\rho+\tau)-f(\rho)}{\mathrm{T}_{\tau}}=2 \mathrm{~S} \cdot \nu \mathrm{U} \tau+\mathrm{T}_{\tau} \cdot f\left(\mathrm{U}_{\tau}\right) .
$$

This is a rigorous equation, connecting the length or the tensor $\mathrm{T}_{\tau}$, of any chord $\tau$ of the ellipsoid, drawn from the extremity of the semidiameter $\rho$, with the direction of that chord $\tau$, or with the versor $U \tau$; it is therefore only a new form of the equation of the ellipsoid itself, with the origin of vectors removed from the centre to a point upon the surface. If we now conceive the chord $\tau$ to diminish in length, the term $\mathrm{T}_{\tau} \cdot f\left(\mathrm{U}_{\tau}\right)$ of the right-hand member of this equation (35.) tends to become $=0$, on account of the factor $T_{\tau}$; and therefore the other term $2 \mathrm{~S} . \nu \mathrm{U}_{\tau}$ of the same member must tend to the same limit zero. In this way we arrive easily at an equation expressing the ultimate law of the directions of the evanescent chords of the ellipsoid, at the extremity of any given or assumed semidiameter $p$; which equation is $0=2 \mathrm{~S} . \nu \mathrm{U}_{\tau}$, or simply,

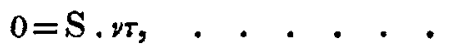

if $\tau$ be a tangential vector. 'The vector $\nu$ is therefore perpendicular to all such tangents, or infinitesimal chords of the ellipsoid, at the extremity of the semidiameter $\rho$; and consequently it has the direction of the normal to that surface, at the extremity of that semidiameter. The tangent plane to the same surface at the same point is represented by the equation (34.), if we treat, therein, the normal vector $y$ as consiant, and if we regard the symbol $\rho$ as denoting, in the same equation (34.), a variable vector, drawn from the centre of the ellipsoid to any point upon that tangent plane. This equation (34.) of the tangent plane may be written as follows:

$$
\text { S. } \nu\left(p-\nu^{-1}\right)=0 ; . . . \quad \cdot .
$$

and under this form it shows easily that the symbol $v^{-1}$ represents, in length and in direction, the perpendicular let fall from the origin of the vectors $p$, that is from the centre of the ellipsoid, upon the plane which is thus represented by the equation (34.) or (37.); so that the vector $v$ itself, as determined by the equation (31.), may be called the vector of proxi- 
mity* of the tangent plane of the ellipsoid, or of an element of that surface, to the centre, at the end of that semidiameter $P$ from which $v$ is deduced by that equation.

46. Conceive now that at the extremity of an infinitesimal chord $\mathrm{d} \rho$ or $\tau$, we draw another normal to the ellipsoid; the expression for any arbitrary point on the former normal, that is the symbol for the vector of this point, drawn from the centre of the ellipsoid, or from the origin of the vectors $\rho$, is of the form $p+n \nu$, where $n$ is an arbitrary scalar; and in like manner the corresponding expression for an arbitrary point on the latter and infinitely near normal, or for its vector from the same centre of the ellipsoid, is $\rho+\mathrm{d} \rho+(n+\mathrm{d} n)(v+\mathrm{d} v)$, where $\mathrm{d} n$ is an arbitrary but infinitesimal scalar, and $\mathrm{d} v$ is the differential of the vector of proximity $v$, which may be found as a function of the differential $\mathrm{d} \rho$ by differentiating the equation (31.), which connects the two vectors $y$ and $\rho$ themselves. In this manner we find, from (31.),

$$
\left(x^{2}-\iota^{2}\right)^{2} \mathrm{~d} \nu=\left(\iota^{2}+x^{2}\right) \mathrm{d} \rho+\imath \mathrm{d} \rho x+x \mathrm{~d} \rho t ; \quad . \quad \text {. }
$$

and the condition required for the intersection of the two near normals, or for the existence of a point common to both, is expressed by the formula

$$
\rho+\mathrm{d} \rho+(n+\mathrm{d} n)(\nu+\mathrm{d} v)=\rho+n v ; . . .
$$

which may be more concisely written as follows:

or thus :

$$
\mathrm{d} \rho+\mathrm{d} . n \nu=0 \text {; . . . . . }
$$

$$
\mathrm{d} \rho+n \mathrm{~d} \nu+\mathrm{d} n \nu=0 . . . \quad \text {. . : }
$$

We can eliminate the two scalar coefficients, $n$ and $\mathrm{d} n$, from this last equation, according to the rules of the calculus of quaternions, by the method exemplified in the 24th article of this paper (Phil. Mag., August 1846), or by operating with the characteristic $S . \nu d v$, because generally

$$
\text { S. } \nu \mu^{2}=0, \quad \text { S. } \nu \mu \nu=0 \text {, }
$$

whatever vectors $\mu$ and $\nu$ may be; so that here,

$$
\mathrm{S} . \nu \mathrm{d} v n \mathrm{~d} v=0, \quad \mathrm{~S} . \nu \mathrm{d} v \mathrm{~d} n v=0 .
$$

"This name, "vector of proximity," was suggested to the writer by a phraseology of Sir John Herschel's; and the equation (31.), of article 45, which determines this vector for the ellipsoid, was one of a few equations which were designed to have been exhibited to the British Association at its meeting in 1846: but were accidentally forwarded at the last moment to Collingwood, instead of Southampton, and did not come to the hands of the eminent philosopher just mentioned, until it was too late for him to do more than return the paper, with some of those encouraging expressions by which he delights to cheer, as ofportunities present themselves, all persons whom he conceives to be labouring usefully for science. 
In this manner we find from (41.) the following very simple formula: S. $\nu \mathrm{d} y \mathrm{~d} \rho=0$;

which is easily seen, on the same principles, to hold good, as the quaternion form of the differential equation of the lines of curvature on a curved surface generally, if $\nu$ be still the vector of proximity of the superficial element of the curved surface to the origin of the vectors $\rho$, which vector $\nu$ is determined by

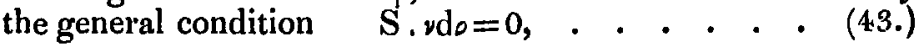
combined with the equation already written,

$$
\text { S. } \nu_{p}=1(34 .) \text {; }
$$

or simply if $v$ be a normal vector, satisfying the condition (43.) alone. Substituting, therefore, in the case of the ellipsoid, the expression for $d \nu$ given by (38.), and observing that S. $v d \rho^{2}=0$, we find that we may write the equation of the lines of curvature for this particular surface as follows :

$$
\text { S. } v(\iota \mathrm{d} \rho x+x \mathrm{~d} \rho) \mathrm{d} \rho=0 ; . . . .
$$

which equation, when treated by the rules of the present calculus, admits of being in many ways symbolically transformed, and may also, with little difficulty, be translated into geometrical enunciations.

47. Thus if we observe that, by article $20, \tau \pi x-x \tau \iota$ is a scalar form, whatever three vectors may be denoted by $b, x, \tau$; and if we attend to the equation (4.3.), which expresses that the normal $\nu$ is perpendicular to the linear element, or infinitesimal chord, $\mathbf{d} \rho$; we shall perceive that, for every direction of that element, the following equation holds good:

$$
\text { S. } v\left(\left(d \mathrm{~d}_{\rho} x-x \mathrm{~d} \rho s\right) \mathrm{d} \rho=0 . \quad . \quad . \quad . \quad\right.
$$

We have therefore, from (44.), for those particular directions which belong to the lines of curvature, this simplified equation;

$$
\text { S. } v \operatorname{d}_{\rho} x \mathrm{~d} \rho=0 ; \quad \text {. . . . . (46.) }
$$

which may be still a little abridged, by writing instead of $\mathrm{d} \rho$ the symbol $\tau$ of a tangential vector, already used in (36.); for thus we obtain the formula:

$$
\text { S. } v i \tau \times \tau=0 . \quad \cdot \quad \cdot \quad \cdot \quad \cdot \quad \cdot(4.7 .)
$$

We might also have observed that by the same article 20 (Phil. Mag., July 1846), $t \tau x+x \tau t$ and therefore $\imath \mathrm{d} \rho x+x \mathrm{~d} \rho t$ is a vector form, and that by article 26 (Phil. Mag., August 1846), three vector-factors under the characteristic $S$ may be in any manner transposed, with only a change (at most) in the positive or negative sign of the resulting scalar; from which it would have followed, by a process exactly similar to the foregoing, that the equation (44.) of the lines of curvature on an ellipsoid may be thus written,

$$
\text { S. } \nu \mathrm{d} \rho \mathrm{d} \rho x=0 \text {; }
$$

Phil. Mag. S. 3. Vol.31. No.208. Oct. 1847. 
or, substituting for the linear element $\mathrm{d} \rho$ the tangential vector $\tau$,

$$
\text { S. } v \tau \imath \tau x=0 ; . . . \cdot . \cdot .
$$

or finally, by the principles of the same 20th article,

$$
\nu \tau i \tau x-x \tau i \tau \nu=0 \text {. . . . . }
$$

48. Under this last form, it was one of a few equations selected in September 1846, for the purpose of being exhibited to the Mathematical Section of the British Association at Southampton; although it happened* that the paper containing those equations did not reach its destination in time to be so exhibited. The equations here marked (49.) and (50.) were however published before the close of the year in which that meeting was held, as part of the abstract of a communication which had been made to the Royal Irish Academy in the summer of that year. (See the Proceedings of the Academy for July 1846, equations (46.) and (47.).) From the somewhat discursive character of the present series of communications on Quaternions, and from the desire which the author feels to render them, to some extent, complete within themselves, or at least intelligible to those mathematical readers of the Philosophical Magazine who may be disposed to favour him with their attention, to the degree which the novelty of the conceptions and method may require, without its being necessary for such readers to refer to other publications of his own, he is induced, and believes himself to be authorized, to copy here a few other equations from that short and hitherto unpublished Southampton paper, and to annex to them another formula which may be found in the Proceedings, already cited, of the Royal Irish Academy : together with a more extensive formula, which he believes to be new.

49. Besides the equation of the ellipsoid,

$$
(i p+p x)(p t+x p)=\left(x^{2}-r^{2}\right)^{2}(21 .), \text { art. 44; }
$$

with the expression derived from it, for the vector of proximity of that surface to its centre,

$$
\left(x^{2}-t^{2}\right)^{2} y=\left(1^{2}+x^{2}\right) p+1 p x+x p r(91 .) \text {, art. } 45 ;
$$

the equation for the lines of curvature on the ellipsoid,

and the equation

$$
\begin{gathered}
v \tau \tau x-x \tau t \tau y=0(50 .), \text { art. } 47 ; \\
v \tau+\tau \nu=0, \cdot \cdot \cdot \cdot .
\end{gathered}
$$

which is a form of the relation $S . y r=0$, that is of the equation (36.), article 45 , of the present series of communications; the author gave, in the paper which has been above referred to, the following symbolic transformation, for the well-known characteristic of operation,

$$
\left(\frac{\mathrm{d}}{\mathrm{d} x}\right)^{2}+\left(\frac{\mathrm{d}}{\mathrm{d} y}\right)^{2}+\left(\frac{\mathrm{d}}{\mathrm{d} z}\right)^{2}
$$

* See the note to article 45 . 
which seems to him to open a wide and new field of analytical research, connected with many important and difficult departments of the mathematical study of nature.

A QUA'TERNION, symbolically considered, being (according to the views originally proposed by the author in 1843) an algebraical quadrinomial of the form $w+i x+j y+k z$, where roxyz are any four real numbers (positive or negative or zero), while $i j k$ are three co-ordinate imaginary units, subject to the fundamental laws of combination (see Phil. Mag. for July 1844):

$$
\left.\begin{array}{l}
i^{2}=j^{2}=k^{2}=-1 ; \\
i j=k ; j k=i ; k i=j ; \\
j i=-k ; k j=-i ; i k=-j ;
\end{array}\right\}
$$

it results at once from these definitions, or laws of symbolic combination, (a.), that if we introduce a new characteristic of operation, $\triangleleft$, defined with relation to these three symbols $i j k$, and to the known operation of partial differentiation, performed with respect to three independent but real variables $x y z$, as follows :

$$
\triangleleft=\frac{i \mathrm{~d}}{\mathrm{~d} x}+\frac{j \mathrm{~d}}{\mathrm{~d} y}+\frac{k \mathrm{~d}}{\mathrm{~d} z} ; . . . \quad . \quad .
$$

this new characteristic $\triangleleft$ roill have the negative of its symbolic square expressed by the following formula:

$$
-\triangleleft^{2}=\left(\frac{\mathrm{d}}{\mathrm{d} x}\right)^{2}+\left(\frac{\mathrm{d}}{\mathrm{d} y}\right)^{2}+\left(\frac{\mathrm{d}}{\mathrm{d} z}\right)^{2} ;
$$

of which it is clear that the applications to analytical physics must be extensive in a high degree. In the paper* designed for Southampton it was remarked, as an illustration, that this result enables us to put the known thermological equation,

$$
\frac{\mathrm{d}^{2} v}{\mathrm{~d} x^{2}}+\frac{\mathrm{d}^{2} v}{\mathrm{~d} y^{2}}+\frac{\mathrm{d}^{2} v}{\mathrm{~d} z^{2}}+a \frac{\mathrm{d} v}{\mathrm{~d} t}=0
$$

under the new and more symbolic form,

$$
\left(\triangleleft^{2}-\frac{a \mathrm{~d}}{\mathrm{~d} t}\right) v=0 ; \quad . \quad . \quad . \quad \text {. }
$$

while $\triangleleft v$ denotes, in quantity and in direction, the $f u x$ of heat, at the time $\ell$ and at the point $x y z$.

50. In the Proceedings of the Royal Irish Academy for July 1846, it will be found to have been noticed that the same new characteristic $\triangleleft$ gives also this other general transformation, perhaps not less remarkable, nor having less extensive

* In that paper itself, the characteristic was written $\nabla$; but this more common sign has been so often used with other meanings, that it seems desirable to abstain fromappropriating it to the new signification here proposed. 
consequences, and which presents itself under the form of a quaternion:

$$
\left.\begin{array}{c}
\triangleleft(i t+j u+k v)=-\left(\frac{\mathrm{d} t}{\mathrm{~d} x}+\frac{\mathrm{d} u}{\mathrm{~d} y}+\frac{\mathrm{d} v}{\mathrm{~d} z}\right) \\
+i\left(\frac{\mathrm{d} v}{\mathrm{~d} y}-\frac{\mathrm{d} u}{\mathrm{~d} z}\right)+j\left(\frac{\mathrm{d} t}{\mathrm{~d} z}-\frac{\mathrm{d} v}{\mathrm{~d} x}\right)+k\left(\frac{\mathrm{d} u}{\mathrm{~d} x}-\frac{\mathrm{d} t}{\mathrm{~d} y}\right)
\end{array}\right\} .
$$

In fact the equations (a.) give generally (see art. 21 of the present series),

$$
\left.\begin{array}{c}
(i x+j y+k z)(i t+j u+k v)=-(x t+y u+z v) \\
+i(y v-z u)+j(z t-x v)+k(x u-y t),
\end{array}\right\} .
$$

if $x y z t u v$ denote any six real numbers; and the calculations by which this is proved, show, still more generally, that the same transformation must hold good, if each of the three symbols $i, j, k$, subject still to the equations (a.), be commutative in arrangement, as a symbolic factor, with each of the three other symbols $x, y, z$; even though the latter symbols, like the former, should not be commutative in that way among themselves; and even if they should denote symbolical instead of numerical multipliers, possessing still the distributive character. We may therefore change the three symbols $x, y, z$, respectively, to the three characteristics of partial differentiation, $\frac{\mathrm{d}}{\mathrm{d} x}, \frac{\mathrm{d}}{\mathrm{d} y}, \frac{\mathrm{d}}{\mathrm{d} z}$; and thus the formula (e.) is seen to be included in the formula (f.). And if we then, in like manner, change the three symbols $t, u, v$, regarded as factors, to $\frac{\mathrm{d}}{\mathrm{d} x^{\prime}}, \frac{\mathrm{d}}{\mathrm{d} y^{\prime}}, \frac{\mathrm{d}}{\mathrm{d} z^{\prime}}$, that is, to the characteristics of three partial differentiations performed with respect to three new and independent variables $x^{\prime}, y^{\prime}, z^{\prime}$, we shall thereby change $\frac{\mathrm{d} t}{\mathrm{~d} x}$ to $\frac{\mathrm{d}}{\mathrm{d} x} \frac{\mathrm{d}}{\mathrm{d} x^{\prime}}$, and so obtain the formula :

$$
\begin{aligned}
& \left(i \frac{\mathrm{d}}{\mathrm{d} x}+j \frac{\mathrm{d}}{\mathrm{d} y}+k \frac{\mathrm{d}}{\mathrm{d} z}\right)\left(i \frac{\mathrm{d}}{\mathrm{d} x^{\prime}}+j \frac{\mathrm{d}}{\mathrm{d} y^{\prime}}+k \frac{\mathrm{d}}{\mathrm{d} z^{\prime}}\right) \\
& \quad=-\left(\frac{\mathrm{d}}{\mathrm{d} x} \frac{\mathrm{d}}{\mathrm{d} x^{\prime}}+\frac{\mathrm{d}}{\mathrm{d} y} \frac{\mathrm{d}}{\mathrm{d} y^{\prime}}+\frac{\mathrm{d}}{\mathrm{d} z} \frac{\mathrm{d}}{\mathrm{d} z^{\prime}}\right) \\
& \quad+i\left(\frac{\mathrm{d}}{\mathrm{d} y} \frac{\mathrm{d}}{\mathrm{d} z^{\prime}}-\frac{\mathrm{d}}{\mathrm{d} z} \frac{\mathrm{d}}{\mathrm{d} y^{\prime}}\right)+j\left(\frac{\mathrm{d}}{\mathrm{d} z} \frac{\mathrm{d}}{\mathrm{d} x^{\prime}}-\frac{\mathrm{d}}{\mathrm{d} x} \frac{\mathrm{d}}{\mathrm{d} z^{\prime}}\right) \\
& \quad+k\left(\frac{\mathrm{d}}{\mathrm{d} x} \frac{\mathrm{d}}{\mathrm{d} y^{\prime}}-\frac{\mathrm{d}}{\mathrm{d} y} \frac{\mathrm{d}}{\mathrm{d} x^{\prime}}\right) ;
\end{aligned}
$$


On the Equation in Numbers $\mathrm{A} x^{3}+\mathrm{B} y^{3}+\mathrm{C} z^{3}=\mathrm{D} x y z$.

which includes the formula (c.), and is now for the first time published.

This formula (g.) is, however, seen to be a very easy and immediate consequence from the author's fundamental equations of 184.3, or from the relations (a.) of the foregoing article, which admit of being concisely summed up in the following continued equation:

$$
i^{2}=j^{2}=k^{2}=i j k=-1 . \text {. . . . . }
$$

The geometrical interpretation of the equation $S . v \tau i x \tau=0$ of the lines of curvature on the ellipsoid, with some other applications of quaternions to that important surface, must be reserved for future articles of the present series, of which some will probably appear in an early number of this Magazine.

[To be continued.]

XLVII. On the Equation in Numbers $\mathrm{A} x^{3}+\mathrm{B} y^{3}+\mathrm{C} z^{3}=\mathrm{D} x y z$, and its associate system of Equations. By J. J. Sylvester, Esq., M.A., F.R.S.*

[Continued from p. 191.]

T $\mathrm{N}$ the last Number of this Magazine I gave an account of a remarkable transformation to which the equation

$$
\mathrm{A} x^{3}+\mathrm{B} y^{3}+\mathrm{C}^{3}=\mathrm{D} x y z
$$

is subject when certain conditions between the coefficients $A$, B, C, D are satisfied; which conditions I shall begin by expressing with more generality and precision than I was enabled to do in my former communication.

1. Two of the quantities $\mathrm{A}, \mathrm{B}, \mathrm{C}$ are to be to one another in the ratio of two cubes.

2. $27 \mathrm{ABC}-\mathrm{D}^{3}$ must contain no positive prime factor whatever of the form $6 n+1$. I erred in my former communication in not excluding cubic factors of this form.

3. If $2^{m}$ is the highest power of 2 which enters into $A B C$, and $2^{n}$ the highest power of 2 which enters into $\mathrm{D}$, then either $m$ must be of the form $3 n \pm 1$, or if not, then $m$ must be greater than $3 n$.

These three conditions being satisfied, the given equation can always be transformed into another,

where

$$
\mathrm{A}^{\prime} u^{3}+\mathrm{B}^{\prime} v^{3}+\mathrm{C}^{\prime} w^{3}=\mathrm{D}^{\prime} u v r w
$$

$$
\mathrm{A}^{\prime} \mathrm{B}^{\prime} \mathrm{C}^{\prime}=\mathrm{ABC} \quad \mathrm{D}^{\prime}=\mathrm{D} \quad u v w=\mathrm{a} \text { factor of } z \text {. }
$$

The consequence of this is, as stated in my former paper, that wherever A, B, C, D, besides satisfying the conditions above stated, are taken so as likewise to satisfy the condition, $1^{\circ}$, of $\mathrm{ABC}$ being equal to $2^{3 m \pm 1}$, or $2^{\mathrm{r}^{\prime}}$, of $\mathrm{ABC}$ being equal to $2^{3 m \pm 1} \cdot p^{3 n \pm 1}$, provided in the first case that $\mathrm{ABC}$ is also of the

* Communicated by the Author. 